\title{
Hardware Platform of Camera-control Part on a Supporting System for Visually Impaired People Operating Touchscreen
}

\author{
Tatsuya Hamachi ${ }^{*}$, Takayoshi Abe, Akira Yamawaki \\ Kyushu Institute of Technology, Fukuoka, Japan (804-8550) \\ *Corresponding Author. yama@ecs.kyutech.ac.jp
}

\begin{abstract}
In recent years, the touchscreen is adopted as various electronic devices. However, on a flat touchscreen, for a vision disabled person, tactile feeling with the finger which is important information may not be obtained. Therefore operativity may be spoiled. We have proposed a wearable supporting system to operate a capacitive touchscreen for them. In a proposal system, an image sensor is equipped under the middle finger and an ear is equipped with the earphone for audio assists. And if a touchscreen is traced by the middle finger, the color barcode which the image sensor caught will be recognized, and the audio assist according to a result will be made. A user pushes an icon not by the middle finger but by an index finger, following an audio assist. In this paper, the hardware platform of the camera -control part in a proposal supporting system is developed. This platform consists of an interface with a camera or a host and an image processing part. The implementation result to FPGA showed that the interface was small and most resources could be used for an image processing part. The image from a camera was able to be displayed on the display through a fundamental verification of operation.
\end{abstract}

Keywords :touchscreen, visually impaired, assist, color barcode, camera

\section{Introduction}

In recent years, the touchscreen is adopted as various electronic devices. However, on a flat touchscreen, for a vision disabled person, tactile feeling with the finger which is important information may not be obtained. Therefore operativity may be spoiled.

Various supporting systems are proposed so far [1-7].
However, there was a problem that operation training was required, a use premise was severe, and it was necessary to replace apparatus. In order to solve such a problem, we have proposed the wearable new touchscreen operational support system supposing an electric capacity type touchscreen [8].

In a proposal system, an image sensor is equipped under the middle finger and an ear is equipped with the earphone for audio assists. Beforehand, the icon displayed on a touchscreen is transposed to the color bar code. A user confirms the position of a touchscreen by touching apparatus using a finger, the body, etc. And if a touchscreen is traced by the middle finger, the color barcode which the image sensor caught will be recognized, and the audio assist according to a result will be made. A user pushes an icon not by the middle finger but by an index finger, following an audio assist. There is sense of security of tracing feeling existence of a touchscreen by a fingertip. Moreover, touchscreen operation can be performed by natural operation.

However, in order to more nearly actually bring close the feeling which traces a touchscreen, it is necessary to shorten distance of a finger and a touchscreen to the utmost. In order to map a photographic subject to up to an image sensor appropriately, it is necessary to separate a lens from a sensor to some extent, and to attach it. In this case, the distance of the middle finger equipped with an image sensor and the index finger which pushes the icon on a touchscreen will spread, and big sense of incongruity will arise in operation. So, distance of a touchscreen and an image sensor is shortened within comfortable limits. If doing it so, the picture of a color barcode will become what the color was mixed and blurred. So, we developed the processing flow which recognizes a color barcode from such a blurred picture, and clarified the construction of a 
color barcode, the number of the color belts which can be used, a fundamental recognition rate, and execution time [9]. Moreover, when the wearable apparatus which is easy to carry is aimed at, execution by an inclusion processor and hardware-ization become indispensable from size, cost, power consumption, etc. So, we developed the hardware of the processing flow shown by literature [9], and it clarified a fundamental recognition rate, performance, and the hardware scale and working speed of hardware [10].

In this paper, The generic hardware platform of a camera-control part is developed in development of a real system. On a proposal platform, image processing hardware is provided with the interface which abstracted the camera and the host. Therefore, if only image processing hardware is rewritten, a camera-control part is realizable. The working speed and the circuit scale of a camera-control part are clarified through the implementation experiment to FPGA.

The rest of the paper is organized as follows. Section 2 describes the outline of a proposal system and camera-control part hardware. Section 3 describes a hardware implementation result. Finally, section 4 concludes our paper.

\section{System Outline}

\subsection{System Configuration}

The composition of a proposal system is shown in Fig. 1 (a). A user equips the point of the middle finger with a camera part, the back of a wrist or a hand with a control part, and an ear with the earphone for audio assists.

A camera part consists of an image sensor and a start button. And a spacer is inserted in order for an image sensor to prevent contacting a touchscreen directly and giving a crack to a screen. Moreover, a soft frame is prepared around a camera part so that a camera part will be covered. Since a camera part is covered by the touchscreen and a frame when a touchscreen is touched through a spacer, the influence of disturbance light is mitigable. When the system wants to operate, contacting the camera part of the middle finger on a touchscreen, a user pushes in the middle finger and pushes a start button. While pushing the start button, a camera part acquires a picture and continues sending it to a control part. In consideration of the size and high-speed communication of image data which are sent to a control part from a camera part, a camera part and a control part are connected with a cable. Since the distance of a camera part

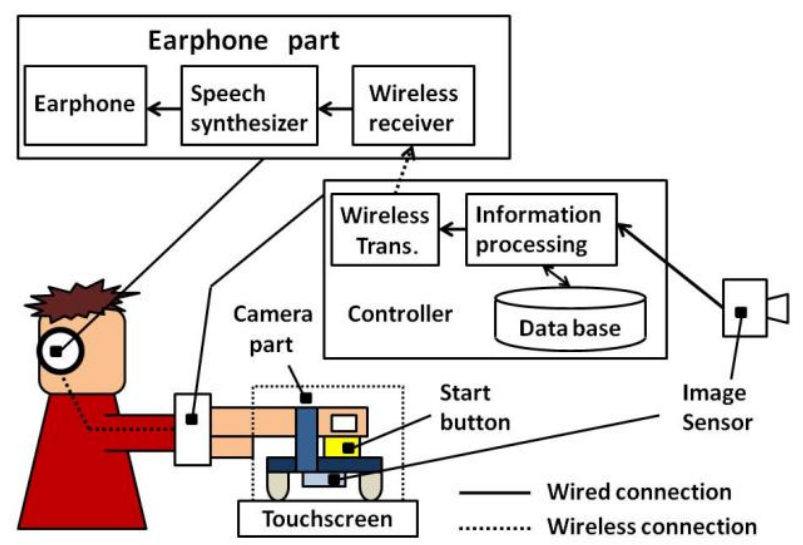

(a) System Overview (Side View)

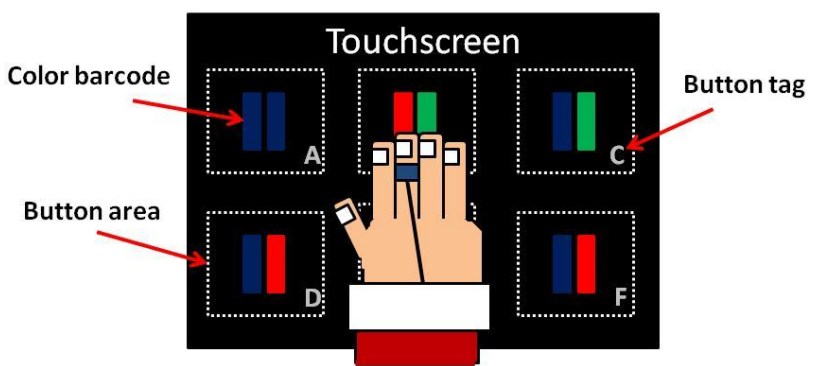

(b) Top view to touchscreen

Fig.1. System Configuration

and a control part is short, even if it connects with a cable, it does not spoil wearable nature.

A control part analyzes the picture sent from the camera part, and recognizes a color barcode. From the recognition result, a control part tells with an audio assist what a user should make to the next. If a user pushes an icon, the below-mentioned button domain containing an icon will change to a specific color, and a control part will recognize that the button was pushed. The control part holds the state of changing according to depression of an icon, and has the database which recorded a state transition diagram, the changes conditions of a state, the screen class and an icon corresponding to them, and data under voice, etc. At the time of depression of an icon, a database is searched and a suitable audio assist is made. The database is rewritable by arbitrary methods. For example, USB memory, SD card, USB, WiFi, Bluetooth, UART, etc.

An audio assist part receives the text data for an audio assist from a control part, and compounds it to a sound in an earphone part. It will decode, if the received text data is enciphered. Since this text data has small size, a control part sends audio assist data to an earphone part by wireless communications. Thereby, although detached and set as compared with a control part and a camera part, a control part and an earphone part can be connected, without 
spoiling wearable nature by wireless connection.

As shown in Fig. 1 (b), a color barcode and a button tag are displayed on the black background color on a touchscreen. A user moves a finger on such a touchscreen, and stops a finger according to an audio assist. However, since a finger does not necessarily exist on a color barcode when a finger is stopped, it corresponds by preparing a button domain. If a finger is in a button domain, a button can be depressed by an index finger. When a healthy person's support is needed, a button tag is used in order to tell the healthy person about what the button on a screen shows.

\subsection{Camera-control part Hardware}

The hardware of Camera part and Controller which were shown in Fig. 1 (a) is developed. The whole circuit composition is as in Fig. 2. This time, processing is not written in Image Processing Hardware. The image from a camera is saved at VRAM without processing and it is displayed on a display from a VGA display part.

The outline of image processing hardware is as follows. The inputs to image processing hardware are a writing place address of the data, a write-in demand signal, and image data from Camera Interface. The writing place address of data is 13 bits, a write-in demand signal is 1 bit, and image data is 16 bits. The outputs from image processing hardware are a writing place address, a write-in demand signal, and image data to VRAM. The writing place address of data is 13 bits, a write-in demand signal is $1 \mathrm{bit}$, and image data is 16 bits.

In the image data of input and output, top 5 bits are B data, following 6 bits are $\mathrm{G}$ data and lower 5 bits are $\mathrm{R}$ data.

By developing hardware in accordance with this specification, image processing becomes possible.

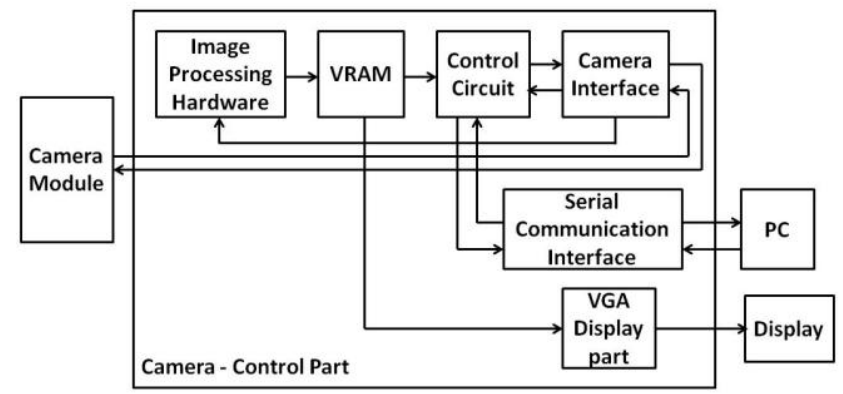

Fig.2. Camera-control part Hardware Configuration

\section{Experiment and Discussion}

\subsection{Experiment Environment}

In this experiment, camera-control part hardware is developed and the working speed and hardware scale are clarified. Experiment environment is shown in Fig. 3 and a source image is shown in Fig. 4. The color barcode of Fig. 4 is taken with the camera of Fig. 3, and it is checked whether the color barcode from a camera is displayed on a display. This time, no processings are added to the acquired color bar code picture. It is necessary to process in an actual proposal system in the picture acquired for color barcode recognition. Fundamental operation of displaying the image from a camera without processing on a display is checked.

\subsection{Implementation Experiment}

The developed camera-control part hardware was implemented in FPGA (Spartan6) of Xilinx. The result is shown in Table 1.

CLK of Table. 1 is the maximum clock frequency that can be inputted. FFs, LUTs, and BRAMs are the number of the circuit resources used within FPGA. FFs corresponds to a Flip-Flop, LUTs corresponds to a Look Up Table, and BRAMs corresponds to an inclusion memory. This result shows using only the $2 \%$ of whole Flip-Flop, using only the $4 \%$ of whole Look Up Table, and using the $25 \%$ of whole inclusion memory. Therefore, most circuit resources can be used for user hardware. Furthermore, Spartan6 used for implementation this time was low-priced edition FPGA,



Fig.3. Experiment Environment



Fig.4. Source Image

Table.1. Result of Implementation on Spartan6

\begin{tabular}{|c|c|c|c|}
\hline CLK $[\mathrm{MHz}]$ & FFs & LUTs & BRAMs \\
\hline 92.6 & $391(2 \%)$ & $393(4 \%)$ & $8(25 \%)$ \\
\hline
\end{tabular}




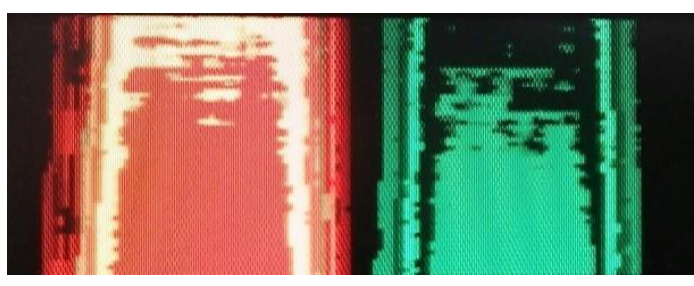

Fig.5. Captured Image

and the scale was third the smallest in a family called Spartan6. In small scale FPGA, since the utilization of circuit resources are $2 \%$ and $4 \%$, a hardware scale is quite small. If implemented in large scale FPGA, the capacity factor of circuit resources becomes small and can use many circuit resources for user hardware.

Moreover, the color bar code was actually taken with the camera, and it was displayed on the display. The display at that time is shown in Fig. 5. This result shows that a red barcode is displayed red and the green barcode is displayed green. Since the image from a camera is displayed on the display, a camera-control part is realizable by rewriting image processing hardware.

\section{Conclusion}

In this paper, the hardware platform of the camera-control part in a proposal supporting system was developed. And it implemented in FPGA and clarified the circuit scale and working speed. The circuit scale was small and it turned out that many circuit resources can be used for an image processing part. Moreover, fundamental operation of displaying the image from a camera on a display has been checked. As a future subject, in a proposal system, since it is necessary to recognize a color barcode tracing a touchscreen, the speed to which a finger is moved, and its angle are verified by processing which is written in an image processing part. And, verification about a blur of acquired picture is performed.

\section{References}

[1] T. Yoshida, K. M. Kitani, H. Koike, S. Belongie, and K. Schlei : "EdgeSonic: Image Feature Sonification for the Visually Impaired," Proc. of the 2nd Augmented Human International Conference pp.11:1-11:4,2011. [2] J.Su, A. Rosenzweig, A. Goel, E. de Lara, and K. N. Truong: "Timbremap: Enabling the Visually-Impaired to Use Maps on Touch-Enabled Devices," Proc. of the 12th International Conference on Human Computer
Interaction with Mobile Devices and Services, pp.17-26, 2010.

[3] G. Yfantidis and G. Evreinov, "Adaptive blind interaction technique for touchscreens," Universal Access in the Information Society, vol.4, no.4, pp.328-337, 2006.

[4] S. K. Kane, J.P. Bigham, and J.O. Wobbrock, "Slide rule: Making Mobile touch screens accessible to blind people using multi-touch interaction technique," Proc. of the ACMSIGACCESS Conference on Computers and Accessibility, pp.73-78, 2008.

[5] T. Handa, T. Sakai, M. Misono, T. Morita, and T. Ito, "Braille presentation method for tactile display," in IEICE Technical Report, WIT200794, 2008, pp.23-26 in japanese.

[6] O. Bau, I. Poupyrev, A. Israr, and C. Harrison, "Teslatouch: Electrovibration for touch surfaces," Proc. of the 23rd ACMSymp. on User Interface Software and Technology, pp.283-292, 2010.

[7] S. Kuno, A. Yamashita, and T. Kaneko, "Assisting System in Touch-Panel Operation Using Stereo Camera," The transactions of the Institute of Electrical Engineers of Japan. D, A publication of Industry Applications Society Vol.131 no. 4, pp.458-465, 2011 in japanese.

[8] A. Yamawaki and S. Serikawa: "A Wearable Supporting System for Visually Impaired People in Operating Capacitive Touchscreen," Applied Mechanics and Materials, Vol.103 pp.687-694, 2011.

[9] A. Yamawaki and S. Serikawa: "A Detection Method of Color Barcode on a Supporting System for Visually Impaired People in Operating a Touchscreen", HCG SYMPOSIUM 2012 pp.159-166, 2012 in japanese.

[10] A. Yamawaki and S. Serikawa : "Development of Image Processing Hardware on a System Supporting Visually Impaired People Using Touchscreen with Finger Camera", The Japanese Journal of the Institute of Industrial Applications Engineers, Vol.1, No.1, pp.10-19, 2013 in japanese. 